\title{
Beim Strafrecht hört die Freundschaft auf
}

\author{
Von RiLG Prof. Dr. Kai Ambos und Dr. Stefanie Bock, Göttingen
}

Beweisverwertung bei Verstoß gegen Belehrungspflichten Rechtskreistheorie - gerichtliche Zuständigkeit in Strafsachen Verlesungs- und Verwertungsverbot des $\$ 252$ StPO - Nachtragsanklage - rechtlicher Hinweis

Der folgende Sachverhalt wurde im Wintersemester 2010/ 2011 an der Georg-August-Universität Göttingen als Abschlussklausur in der StPO-Vorlesung ausgegeben. Die als leicht bis mittel-schwer einzustufende Zwischenprüfungsklausur ist sehr gut ausgefallen, der Notendurchschnitt lag bei 8,3 Punkten, die Durchfallquote bei unter 5\%. Die Bewertung der Arbeiten orientierte sich an folgenden Grund- sätzen: Insgesamt konnten maximal 54 Punkte erreicht werden. Dabei entfielen 48 Punkte auf die Beantwortung der Fragen (zur genauen Aufteilung s. Lösungsskizze), die verbleibenden sechs Punkte auf den Gesamteindruck, insbesondere Argumentationsvermögen der Bearbeiter. Die erzielte Punktzahl geteilt durch drei ergab die Gesamtnote.

\section{SACHVERHALT}

Der in Herzberg wohnende, nicht vorbestrafte A wird verdächtigt, gemeinsam mit seinem Freund F in Göttingen am 3. 1. 2011 den $\mathrm{O}$ zusammengeschlagen und ihm $100 €$ weggenommen zu 
haben. Gegenüber der Polizei sagt der ordnungsgemäß belehrte A aus, er sei zur Tatzeit bei seiner Ehefrau E gewesen. Diese wird daraufhin zur polizeilichen Zeugenvernehmung vorgeladen.

Frage 1: Muss E der polizeilichen Vorladung Folge leisten? Begründen Sie Ihre Antwort!

Nach einem heftigen Streit mit A geht E zur Polizei und erzählt dort - nach ordnungsgemäßer Belehrung - dem Polizeibeamten P, dass A am 3. 1. 2011 den ganzen Tag mit F unterwegs gewesen und erst spät in der Nacht nach Hause gekommen sei. Die Aussage wird protokolliert. P befragt daraufhin den F, ohne diesen aber auf sein Schweigerecht hinzuweisen. F legt ein volles Geständnis ab und belastet dabei auch den A schwer: Dieser habe den Vorschlag gemacht, den $\mathrm{O}$ »abzuziehen « und auch einen Großteil der Beute behalten.

Frage 2: Ist die Aussage des F im Verfahren gegen A verwertbar?

Die Akten werden dem zuständigen Staatsanwalt S vorgelegt. Dieser will nun Anklage gegen A wegen Raubes in Tateinheit mit Körperverletzung erheben.

Frage 3: Welcher Tatverdacht ist hierfür erforderlich? Definieren Sie diesen!

Frage 4: Abgesehen von der Einreichung einer Anklage gem. $\S 170$ I StPO: wie kann das Ermittlungsverfahren noch beendet werden?

Frage 5: Vor welchem Gericht wird S Klage erheben?

Bearbeitervermerk: Herzberg und Göttingen haben jeweils ein Amtsgericht, die beide im Bezirk des Landgerichts Göttingen liegen. Dieses gehört wiederum zum Oberlandesgericht Braunschweig.

In der Hauptverhandlung wird die E als Zeugin gehört. Da sie sich mittlerweile wieder mit A versöhnt hat, beruft sie sich auf ihr Zeugnisverweigerungsrecht. Unter Protest von A und seinem Verteidiger beantragt $S$ daraufhin, das Protokoll ihrer polizeilichen Vernehmung zu verlesen, hilfsweise den Polizisten $P$ als Zeugen zu vernehmen, damit dieser aussagt, dass die $\mathrm{E}$ in ihrer ersten Vernehmung das (vorgebliche) Alibi des A nicht bestätigt habe.

Frage 6: Wie wird das Gericht entscheiden?

Als nächstes wird $O$ in den Zeugenstand gerufen. Dieser identifiziert A als einen der beiden Angreifer, die ihn »abgezogen « haben. Dabei erwähnt O erstmalig, dass A bei der Tat griffbereit ein großes Messer im Hosenbund stecken hatte. Zudem berichtet $\mathrm{O}$, dass A zwei Tage vor der Hauptverhandlung $\mathrm{zu}$ ihm gekommen sei und ihm gedroht habe, er würde ihn krankenhausreif schlagen, wenn er (der O) seine Beschuldigungen vor Gericht aufrecht erhalten würde.

Frage 7: Wie werden bzw. können das Gericht und/oder S auf diese neuen Informationen reagieren?

A wird zu einer hohen Freiheitsstrafe ohne Bewährung verurteilt.

Frage 8: Welche Möglichkeiten hat A, gegen das Urteil vorzugehen?

\section{LÖSUNG}

Frage 1: Muss E der polizeilichen Vorladung Folge leisten? (4 Punkte)

Gem. $\$ 163$ III $^{1}$ ist die Polizei zur Vernehmung von Zeugen befugt. Darüber hinaus hat sie das gesetzlich nicht ausdrücklich geregelte Recht, Zeugen vorzuladen ${ }^{2}$. Dabei handelt es sich allerdings lediglich um eine unverbindliche Aufforderung. Es besteht keine Pflicht vor der Polizei zu erscheinen ${ }^{3}$. Dies ergibt sich aus folgender, gesetzessystematischer Erwägung: Gem. §48 I 1 sind Zeugen verpflichtet, zu dem zu ihrer Vernehmung bestimmten Termin vor dem Richter zu erscheinen. Gleiches gilt gem. $\S 161$ a I 1 bei einer Vorladung durch die Staatsanwaltschaft. Für polizeiliche Vernehmungen fehlt hingegen eine entsprechende Regelung. Der insoweit einschlägige $\S 163$ III verweist nur auf $\S 161$ a III 2-4, nicht aber auf den die Erscheinenspflicht regelnden Abs. 1.

Anmerkung: Von der Pflicht zum Erscheinen ist die Pflicht zur Aussage zu unterscheiden. Allein die Tatsache, dass der E als Ehefrau gem. \$5 I Nr. 2 ein Zeugnisverweigerungsrecht zusteht, würde sie nicht von ihrer Pflicht zum Erscheinen entbinden. Dies gilt selbst dann, wenn sie bereits angekündigt hätte, von ihrem Zeugnisverweigerungsrecht $\mathrm{Ge}$ brauch machen zu wollen ${ }^{4}$

Frage 2: Ist die Aussage des F im Verfahren gegen A verwertbar? (9 Punkte)

Gem. § 136 I 2 ist der Beschuldigte bei seiner ersten Vernehmung darüber zu belehren, dass es ihm freisteht, sich zur Sache einzulassen oder zu schweigen. Dies gilt gem. § 163 a IV 2 auch bei einer polizeilichen Befragung. Zum Zeitpunkt der Vernehmung des F kam dieser bereits ernstlich als Täter des Raubes an $\mathrm{O}$ in Betracht und war daher in jedem Fall als Beschuldigter zu belehren $^{5}$. Da P dies nicht getan hat, liegt ein Verstoß gegen $\S 136$ I 2 i. V. m. § 163 a IV 2 vor. Dies macht die Aussage grundsätzlich unverwertbar ${ }^{6}$. Fraglich ist aber, ob dies auch im Verfahren gegen A gilt. Nach der (ursprünglich) vom BGH vertretenen Rechtskreistheorie hängt die Verwertbarkeit von Beweisen davon ab, ob die Verletzung der strafprozessualen Norm den Rechtskreis des Beschuldigten wesentlich berührt oder ob sie für ihn von untergeordneter oder von keiner Bedeutung ist ${ }^{7}$. Nachdem dieser Ansatz auf heftige Kritik in der Literatur stie ${ }^{8}$, stellt der BGH nunmehr zumeist relativierend auf den Verfahrenszweck einer Norm und die Auswirkung ihrer Verletzung auf die Rechtsstellung des Beschuldigten im jeweiligen Einzelfall $\mathrm{ab}^{9}$. Damit nähert sich der BGH der Lehre vom

$1 \S \S$ ohne Nennung sind solche der StPO.

2 Eisenberg Beweisrecht der StPO, 7. Aufl. 2011, Rdn. 1083.

3 BGH NJW 1962, 1020 (1021). Vgl. auch KK-StPO/Griesbaum § 163 Rdn. 15; Kühne Strafprozessrecht, 8. Aufl. 2010, Rdn. 354.

4 Eisenberg Beweisrecht der StPO, 7. Aufl. 2011, Rdn. 1055; Krey Deutsches Strafverfahrensrecht - Band 2, 2007, Rdn.832; Kühne Strafprozessrecht, 8. Aufl. 2010, Rdn. 811; Roxin/Schünemann Strafverfahrensrecht, 26. Aufl. 2009, § 26 Rdn. 11.

5 Vgl. BGHSt 37, 48 (51 f.) sowie KK-StPO/Diemer $\$ 136$ Rdn. 4. Vgl. hierzu auch Murmann Prüfungswissen Strafprozessrecht, 2. Aufl. 2010, Rdn. $209 \mathrm{f}$.

6 BGHSt 38, 214; BGH NStZ 2007, 653; Schroeder/Verrel Strafprozessrecht 5. Aufl. 2011, Rdn. 136; Ambos Beweisverwertungsverbote, 2010, 52 m. w. N. $\mathrm{Zu}$ den - hier nicht einschlägigen - Ausnahmen vgl. Volk Grundkurs StPO, 7. Aufl. 2010, §9 Rdn. 11; Kindhäuser Strafprozessrecht, 2. Aufl. 2010, §6 Rdn. 50. Insbesondere zur umstrittenen Widerspruchslösung des BGH, wonach eine Verwertung der Aussage zulässig ist, wenn der Verteidiger des Beschuldigten der Verwertung zustimmt oder ihr nicht rechtzeitig und begründet widerspricht, BGHSt 38, 214; 42, 15 (22f.); 52, 38; ebenso Volk a. a. O.; a. A. Roxin/ Schünemann Strafverfahrensrecht, 26.Aufl. 2009, §24 Rdn.34; Beulke Strafprozessrecht, 11. Aufl. 2010, Rdn. 150 m.w. N.; kritisch auch Murmann Prüfungswissen Strafprozessrecht, 2. Aufl. 2010, Rdn. 214, vertiefend Heinrich ZStW 112 (2000), 398.

7 Grundlegend BGHSt 11, 213 (215)

8 Gegen die Rechtskreistheorie wird insbesondere ihre mangelnde Bestimmtheit ins Feld geführt. Darüber hinaus führt sie zu einer Beschränkung der Verfahrensrechte des Angeklagten auf wesentliche Rechte, obwohl er doch einen Anspruch auf ein generell justizförmiges Verfahren hat (vgl. auch die Nachweise in Fn. 12) und nicht nur die Verletzung seines Rechtskreises zu einem Verwertungsverbot führen kann. Schließlich vermag die »Rechtskreisberührung « nichts über deren Folgen mit Blick auf die Beweisverwertung auszusagen; vgl. Ambos Beweisverwertungsverbote, 2010, $41 \mathrm{f}$. m. w. N.

9 Vgl. hierzu den Rechtsprechungsüberblick bei Kühne Strafprozessrecht, 8. Aufl. 2010, Rdn. 98; s. aber auch die Entscheidung BGHSt 53, 191, in der der BGH 
Schutzzweck der Norm an, die danach fragt, ob Sinn und Zweck einer verletzten Verfahrensvorschrift ein Verwertungsgebot gebieten ${ }^{10}$.

Im vorliegenden Fall ließe sich wie folgt argumentieren: Die Belehrungspflicht des $§ 136$ I 2 bezweckt ausschließlich den Schutz des jeweils betroffenen Beschuldigten (in casu den Schutz des F), dient aber nicht den Interessen von Mitbeschuldigten (in casu den Interessen von A). Deren Rechtskreis wird von einem gegen andere Beschuldigte gerichteten Verstoß gegen die Belehrungsvorschrift nach $§ 136$ I 2 grundsätzlich nicht berührt ${ }^{11}$. Demnach wäre die Aussage des F im Verfahren gegen A verwertbar. Hiergegen lässt sich allerdings anführen, dass $\mathrm{F}$ ein Recht darauf hat, dass nur justizförmig gewonnene Beweismittel gegen ihn verwendet werden ${ }^{12}$.

Anmerkung: Mit entsprechender Begründung sind beide Ansichten gut vertretbar. Entscheidend ist, dass die Bearbeiter das Problem erkennen und sich hiermit argumentativ auseinandersetzen.

Frage 3: Welcher Tatverdacht ist hierfür erforderlich? Definieren Sie diesen! (3 Punkte)

Gem. § 170 Ii. V. m. § 203 erhebt die Staatsanwaltschaft öffentliche Klage, wenn ein hinreichender Tatverdachtbesteht. Diesist dann der Fall, wenn bei vorläufiger Tatbewertung die Verurteilung des Beschuldigten mit Wahrscheinlichkeit zu erwarten ist ${ }^{13}$. Eshandeltsichalso um einein die Zukunftgerichtete Prognoseentscheidung der Staatsanwaltschaft ${ }^{14}$. Es wird gerade nicht vergangenheitsbezogen gefragt, ob es wahrscheinlich ist, dass der Beschuldigte die ihm zur Last gelegte Tat begangen hat.

Frage 4: Abgesehen von der Einreichung einer Anklage gem. $\S 170$ I: wie kann das Ermittlungsverfahren noch beendet werden? (3 Punkte)

Kommt die Staatsanwaltschaft nach Abschluss der Ermittlungen zu dem Ergebnis, dass kein hinreichender Tatverdacht besteht, so ist das Verfahren gem. $§ 170$ II einzustellen. Anderenfalls kann sie - alternativ zur Einreichung der Anklage bei Gericht - den Erlass eines Strafbefehls beantragen (§407 I) oder das Verfahren aus Opportunitätsgründen einstellen ( $153 \mathrm{ff}$. $)^{15}$.

Anmerkung: Darüber hinaus existieren noch weitere besondere Formen der Anklageerhebung, z. B. der Antrag auf Aburteilung im beschleunigen Verfahren (\$ 417), der Antrag im vereinfachten Jugendstrafverfahren ( $\$ 76 \mathrm{JGG})$, und der Antrag im Sicherungsverfahren bei Schuldlosigkeit des Betroffenen gem. §20 StGB (§413). Die Kenntnis dieser Formen der Anklageerhebung wurde von den Studierenden nicht verlangt; wurden sie dennoch genannt, wurde dies positiv vermerkt.

Frage 5: Vor welchem Gericht wird S Klage erheben? (6 Punkte)

\section{a) Sachliche Zuständigkeit}

Die sachliche Zuständigkeit der Strafgerichte ist im GVG geregelt $^{16}$. Das Amtsgericht ist gem. $§ 24$ I GVG zuständig, wenn nicht

- die zwingende Zuständigkeit des Landgerichts oder des Oberlandesgericht begründet ist oder

- eine höhere Strafe als vier Jahre Freiheitsstrafe zu erwarten ist oder

- die Staatsanwaltschaft wegen der besonderen Schutzbedürftigkeit von Verletzten der Straftat, die als Zeugen in Betracht kommen, des besonderen Umfangs oder besonderen Bedeutung des Falles Anklage beim Landgericht erhebt.

Bei Raub (§ 249 I StGB) und Körperverletzung ( 223 I StGB) handelt es sich weder um Kapitaldelikte gem. § 74 II GVG, die in die Zuständigkeit des landgerichtlichen Schwurgerichts fallen würden, noch um Staatsschutzsachen i. S. d. $\$ \S 74$ a, 120
GVG, die vor dem Landgericht bzw. dem Oberlandesgericht verhandelt werden müssten. Da A nicht vorbestraft und der eingetretene Schaden gering ist, ist die auf vier Jahre begrenzte Strafgewalt des Amtsgerichts jedenfalls ausreichend. Anlass, ausnahmsweise Anklage vor dem Landgericht zu erheben, besteht nicht. Zuständig ist daher das Amtsgericht.

Das Amtsgericht besteht aus zwei Spruchkörpern: dem Strafrichter und dem Schöffengericht. Ersterer entscheidet über Privatklagen und Vergehen, bei denen nicht mehr als zwei Jahre Freiheitsstrafe zu erwarten sind ( $\$ 25$ GVG). Der Raub ist im Mindestmaß mit einer Freiheitsstrafe von einem Jahr bedroht. A wird damit gem. §12 I StGB eines Verbrechens beschuldigt. Die Tatsache, dass im vorliegenden Fall u. U. eine Bestrafung wegen Raubes im minder schweren Fall gem. §249 II StGB in Betracht kommt, ändert nichts an der Einordnung der Tat als Verbrechen (§ 12 III StGB). Es ist daher die Zuständigkeit des Schöffengerichts eröffnet, ohne dass es auf die Höhe der im konkreten Fall zu erwartenden Strafe ankäme.

\section{b) Örtliche Zuständigkeit}

Die örtliche Zuständigkeit richtet sich sowohl nach dem Tatort ( 77 - in casu Göttingen) als auch dem Wohnsitz des Angeschuldigten (\$8-in casu Herzberg). Sind, wie im vorliegenden Fall, mehrere Gerichte örtlich zuständig, kann die Staatsanwalt wählen, bei welchem sie Anklage erhebt ${ }^{17}$. S kann die Anklage also entweder an das Schöffengericht Herzberg oder das Schöffengericht Göttingen richten.

Frage 6: Wie wird das Gericht entscheiden? (11 Punkte)

Gem. §244 III 1 ist ein Beweisantrag abzulehnen, wenn die Erhebung des Beweises unzulässig ist.

\section{a) Verlesung des polizeilichen Protokolls}

Einer Verlesung des polizeilichen Protokolls könnte der Grundsatz der persönlichen Vernehmung entgegenstehen. Gem. §250 müssen Wahrnehmungen von Zeugen und Sachverständigen durch ihre Vernehmung in den Prozess eingeführt werden. Der Personalbeweis darf grundsätzlich nicht durch die Verlesung von Protokollen ersetzt werden. Dies ist Ausdruck des Unmittelbarkeitsgrundsatzes ${ }^{18}$. Ausnahmen sind nur unter den in $§ 251 \mathrm{I}^{19}$ genannten Voraussetzungen möglich. Nach

weiterhin (zumindestergänzend) auf die Rechtskreistheorie abstellt; hierzuauch Beulke Strafprozessrecht, 11. Aufl. 2010, Rdn. 459; Gless NStZ 2010, 98.

10 Vgl. Roxin/Schünemann Strafverfahrensrecht, 26. Aufl. 2009, § 24 Rdn. 25; Arloth GA 2006, 259 (260); Ambos Beweisverwertungsverbote, 2010, 42.

11 BGH StV 1995, 231 (232) mit abl. Anmerkung Dencker; BayObLG StV 1995 237; ähnlich auch BGHSt 53, $191 \mathrm{~m}$. Anm. Gless NStZ 2010, 98. Vgl. auch Ambos Beweisverwertungsverbote, 2010, 55 m.w. N

12 Roxin/Schünemann Strafverfahrensrecht, 26. Aufl. 2009, §24 Rdn. 32; ähnlich auch LR/Gless $§ 136$ Rdn. 90. Vgl. auch Kindhäuser Strafprozessrecht, 2. Aufl. 2010, §23 Rdn. 13.

13 Volk Grundkurs StPO, 7. Aufl. 2010, § 8 Rdn. 3; Meyer-Goßner § 170 Rdn. 1 m.w. N.

14 Roxin/Schünemann Strafverfahrensrecht, 26. Aufl. 2009, § 40 Rdn. 2.

15 Vgl. hierzu auch den Überblick bei Beulke Strafprozessrecht, 11. Aufl. 2010, Rdn. 319-320.

16 Siehe hierzu auch Volk Grundkurs StPO, 7. Aufl. 2010, §5 Rdn. 6 ff.; Beulke Strafprozessrecht, 11. Aufl. 2010, Rdn. 39f.; Kühne Strafprozessrecht, 8. Aufl. 2010, Rdn. $125 \mathrm{ff}$

17 Meyer-Goßner Vor $\S 7$ Rdn. 10; Volk Grundkurs StPO, 7. Aufl. 2010, §5 Rdn. 16.

18 BGHSt 51, 325 (328); Volk Grundkurs StPO, 7. Aufl. 2010, § 27 Rdn. 1; Beulke Strafprozessrecht, 11. Aufl. 2010, Rdn. 410.

19 Abs. 2 bezieht sich auf den hier nicht einschlägigen Fall einer richterlichen Vernehmung. 
Ansicht des BGH ist dieser allerdings nur dann anwendbar, wenn der Zeuge in der Hauptverhandlung nicht erscheint, nicht aber wenn er - wie die E hier - Angaben zur Person macht und anschließend die Aussage zur Sache verweigert ${ }^{20}$.

Anmerkung: Zweifelhaft erscheint zudem, ob eine der in § 251 I Nr. 1 genannten Ausnahmekonstellationen vorliegt. Da A und sein Verteidiger bereits gegen den Beweisantrag protestiert haben, kommt eine einverständliche Verlesung des Protokolls nach $§ 251$ I Nr. 1 nicht in Betracht. Darüber hinaus kann gem. §251 INr. 2 auch dann eine Ausnahme vom Unmittelbarkeitsgrundsatz gemacht werden, wenn der Zeuge verstorben ist oder aus einem anderen Grunde in absehbarer Zeit gerichtlich nicht vernommen werden kann. Hier steht der Vernehmung der E ein rechtliches Hindernis - nämlich ihre Berufung auf ihr Aussageverweigerungsrecht - entgegen. Ob in solchen Konstellationen eine Protokollverlesung zulässig sein kann, ist umstritten. Hiergegen spricht, dass § 251 I 1 Nr. 2 voraussetzt, dass der Zeuge nicht vernommen werden kann. Im Falle eines Zeugnis- oder Auskunftsverweigerungsrechts muss der Zeuge allerdings zur Person aussagen und das Bestehen seiner Rechtsposition glaubhaft machen ${ }^{21}$. Daher wird man wohl davon ausgehen müssen, dass eine Protokollverlesung nur in Betracht kommt, wenn die Vernehmung des Zeugen aus tatsächlichen Gründen unmöglich ist, nicht aber wenn ihr nur rechtliche Hindernisse entgegenstehen ${ }^{22}$.

Selbst wenn die Voraussetzungen des $§ 251$ I erfüllt wären, ist jedenfalls das Verlesungsverbot des $\$ 252 \mathrm{zu}$ beachten ${ }^{23}$. Hiernach dürfen Aussagen eines vor der Hauptverhandlung vernommenen Zeugen, der sich erst in der Hauptverhandlung auf sein Zeugnisverweigerungsrecht ${ }^{24}$ beruft, nicht verlesen werden. Dies dient dem Schutz der in $§ 52 \mathrm{ff}$. genannten Rech$t^{25}$. Da E als Ehefrau des Angeklagten berechtigterweise die Aussage verweigert ( 552 I Nr. 2), wird das Gericht den Antrag auf Verlesung des polizeilichen Protokolls wegen Verstoßes gegen $§ 252$ ablehnen.

\section{b) Vernehmung der Verhörsperson P}

Fraglich ist, ob die Vernehmung der Verhörsperson P zulässig ist. Seinem Wortlaut nach untersagt $\$ 252$ lediglich die Verlesung des polizeilichen Protokolls. Allein die Tatsache, dass $P$ nur Zeuge vom Hörensagen wäre, steht seiner Vernehmung nicht entgegen ${ }^{26}$. Würde man $\$ 252$ allerdings als reines Verlesungsverbot ansehen, bliebe ihm kein/kaum ein eigenständiger Anwendungsspielraum gegenüber $§ 250$ S. $2^{27}$. Zudem soll $\S 252$ gewährleisten, dass der Zeugnisverweigerungsberechtigte bis zur Hauptverhandlung frei entscheiden kann, ob seine frühere, vielleicht voreilig gemachte Aussage verwertet werden darf $^{28}$. Diese Zielsetzung würde konterkariert, wenn die frühere Aussage über die Vernehmung der Verhörsperson eingeführt werden könnte ${ }^{29}$. 252 ist daher ein umfassendes Verwertungsverbot zu entnehmen ${ }^{30}$. Untersagt ist nicht nur die Verlesung des Protokolls einer früheren Vernehmung, sondern auch jede Einführung des Aussageinhalts in die Hauptverhandlung, etwa durch Vernehmung der Verhörsperson ${ }^{31}$. Die Rechtsprechung macht hiervon lediglich dann eine Ausnahme, wenn der Zeuge vor der Hauptverhandlung von einem Richter vernommen wur$\mathrm{de}^{32}$. Damit steht $§ 252$ auch der Vernehmung des P entgegen. Das Gericht wird den Beweisantrag der Staatsanwaltschaft daher insgesamt ablehnen.

Frage 7: Wie werden bzw. können das Gericht und/oder S auf diese neuen Informationen reagieren? (10 Punkte)

Wenn A beim Überfall auf $O$ griffbereit ein großes Messer bei sich geführt hat, so hätte er sich wegen schweren Raubes gem. $\S 250$ I Nr. 1 a) StGB strafbar gemacht - die Anklage lautet aber nur auf einfachen Raub. Zudem gibt die Aussage des O Anlass zur Annahme, dass A sich ferner wegen versuchter Nötigung gem. §240 I, III StGB und versuchter Anstiftung zur Falschaussage gem. $§ 159$ StGB strafbar gemacht hat.

\section{a) Rechtlicher Hinweis versus Nachtragsanklage}

Tauchen in der Hauptverhandlung neue tatsächliche oder rechtliche Aspekte auf, können sie entweder durch einen rechtlichen Hinweis (§265 I) oder durch eine Nachtragsanklage $(\$ 266)$ in das Verfahren eingebracht werden. Welche Vorgehensweise die richtige ist, entscheidet sich danach, ob die neuen Erkenntnisse die angeklagte Tat betreffen oder ob sie eine eigenständige Tat darstellen. Maßgeblich ist also der Begriff der Tat im prozessualen Sinne ${ }^{33}$. Hierunter versteht man einen einheitlichen geschichtlichen Vorgang, das gesamte Verhalten des Täters, soweit es nach natürlicher Auffassung einen einheitlichen Lebenssachverhalt darstellt ${ }^{34}$. Anders ausgedrückt liegt eine einzige prozessuale Tat immer dann vor, wenn die getrennte Aburteilung der einzelnen Handlungen als unnatürliche Aufspaltung eines einheitlichen Lebensvorgangs empfunden würde ${ }^{35}$.

\section{b) Das Beisichführen eines Messers}

Die Aussage des O, der A habe ein Messer bei sich geführt, bezieht sich auf den angeklagten Sachverhalt: nämlich den Überfall auf O durch A und F in Göttingen am 3.1.2011. Es

20 BGH NStZ 1982, 342; vgl. auch BGHSt 51, 325 (329); Geppert JURA 1988, 305 (307); Volk Grundkurs StPO, 7. Aufl. 2010, § 27 Rdn. 2.

21 Ausführlich BGHSt 51, 325 (330 ff.).

22 BGHSt 51, 325; Cornelius NStZ 2008, 244 (247); Meyer-Goßner § 251 Rdn. 11. A. A. Hecker JR 2008, 121; Murmann StV 2008, 339 (340 f.).

23 Vertiefend Geppert JURA 1988, 305 und 363; Kraatz JURA 2011, 170

24 Der Streit, ob \$251 I Nr. 2 eine Protokollverlesung bei rechtlichen Hindernissen gestattet, ist daher in erster Linie relevant, wenn sich der Zeuge in der Hauptverhandlung auf sein Auskunftsverweigerungsrecht nach $\S 55$ beruft, da dieses nach h. M. nicht dem Anwendungsbereich des $\$ 252$ unterfällt, vgl. Meyer-Goßner $\$ 252$ Rdn. 5 m. w. N. (auch zur Gegenauffassung); a. A. z. B. Kühne Strafprozessrecht, 8. Aufl. 2010, Rdn. 943.

25 KK/Diemer $\$ 252$ Rdn. 1; Geppert JURA 1998, 305 (306); Kraatz JURA 2011, 170 .

26 Geppert JURA 1988, 305 (307); Kraatz JURA 2011, 170 (171); Kühne Strafprozessrecht, 8. Aufl. 2010, Rdn. 915; Murmann Prüfungswissen Strafprozessrecht, 2. Aufl. 2010, Rdn. 248 f.; Volk Grundkurs StPO, 7. Aufl. 2010, § 27 Rdn. 28.

27 Geppert JURA 1988, 305 (307).

28 BGHSt 45, 203 (208). Vgl. auch BVerfG NStZ-RR 2004, 18 (19).

29 BVerfG NStZ-RR 2004, 18 (19); Geppert JURA 1988, 305 (307); Kraatz JURA 2011, 170 (171).

30 Pfeiffer StPO, §252 Rdn. 1; KK/Diemer §252 Rdn. 1; Kühne Strafprozessrecht, 8. Aufl. 2010, Rdn. 943; Kraatz JURA 2011, 170 (171).

31 BGHSt 20, 384 (386)

32 Grundlegend BGHSt 2, 99 (106); auch BGHSt 49, 72 (77); zustimmend Krey Deutsches Strafverfahrensrecht - Band 2, 2007, Rdn. 1015; Kühne Strafprozessrecht, 8. Aufl. 2010, Rdn. 945; Schroeder/Verrel Strafprozessrecht, 5. Aufl. 2011, Rdn. 257. Zur Begründung wird vor allem auf die höhere Qualität der richterlichen gegenüber der staatsanwaltschaftlichen bzw. polizeilichen Vernehmung verwiesen. Dies vermag allerdings hinsichtlich der staatsanwaltschaftlichen o. richterlichen Vernehmung nicht zu überzeugen. Wenn man auf die Aussage des Zeugen aus dem Ermittlungsverfahren nicht verzichten will, muss man $§ 252$ eng i. S. eines bloßen Vernehmungsverbotes auslegen, nicht aber nicht belegbare Qualitätsunterschiede von - durch Volljuristen durchgeführte - Beschuldigtenvernehmungen unterstellen, vgl. Ambos Beweisverwertungsverbote, 2010, 37 m. w. N.; kritisch auch Beulke Strafprozessrecht, 11. Aufl. 2010, Rdn. 420; vgl. zur Vernehmung richterlicher Vernehmungspersonen auch Kraatz JURA 2011, 170 (175f.).

33 Vgl. auch Volk Grundkurs StPO, 7. Aufl. 2010, § 13 Rdn. 3; Beulke Strafprozessrecht, 11. Aufl. 2010, Rdn.385; Kindhäuser Strafprozessrecht, 2. Aufl. 2010, § 25 Rdn. 9-10.

34 Beulke Strafprozessrecht, 11. Aufl. 2010, Rdn.513; Volk Grundkurs StPO, 7. Aufl. 2010, $\S 13$ Rdn. 2 beide m. w. N.; vertiefend Kindhäuser Strafprozessrecht, 2. Aufl. 2010, §25 Rdn. $12 \mathrm{ff}$.

35 BGHSt 49, 359 (362); Krey Deutsches Strafverfahrensrecht - Band 2, 2007, Rdn. 1169; Beulke Strafprozessrecht, 11. Aufl. 2010, Rdn. 513 m.w. N. 
handelt sich daher um dieselbe prozessuale Tat ${ }^{36}$. Dass das Gericht diese neue Tatsache berücksichtigen darf, ergibt sich bereits aus $\$ 264$ II, demzufolge das Gericht nicht an die Beurteilung der Tat, die dem Beschluss über die Eröffnung des Hauptverfahrens zugrunde liegt, gebunden ist. Um den Angeklagten aber vor Überraschungen zu schützen und ihm eine effektive Verteidigung zu ermöglichen ${ }^{37}$, ist er gem. § 265 I auf die Veränderung des rechtlichen Gesichtspunktes hinzuweisen. Der vorsitzende Richter wird den A daher darüber informieren, dass aufgrund der Aussage des $\mathrm{O}$ nunmehr auch eine Verurteilung wegen schweren Raubes gem. § 250 I Nr. 1 a) StGB in Betracht kommt. S muss nicht weiter tätig werden.

Anmerkung: Auch wenn schwerer Raub mit einer Mindestfreiheitsstrafe von drei Jahren bedroht ist, ist mit Blick auf die niedrige Schadenshöhe und unter Berücksichtigung der Tatsache, dass A nicht vorbestraft ist, die Strafgewalt des Amtsgerichts (Freiheitsstrafe bis zu vier Jahren, siehe oben Frage 5) ausreichend. Eine Verweisung der Sache an das Landgericht gem. §270 ist daher nicht erforderlich.

\section{c) Der Einschüchterungsversuch}

Der Einschüchterungsversuch des A fand an einem anderen Ort und zu einer anderen Zeit statt als der angeklagte Überfall. Zudem ist das Tatbild ein völlig anderes. Daher handelt es sich um eine neue prozessuale Tat, die bisher nicht angeklagt wurde. Damit darf sich das Gericht grundsätzlich mit der Bedrohung des $\mathrm{O}$ nicht befassen, da es nach dem Anklagegrundsatz (\$151) nur über Tatsachen befinden kann, die von der Staatsanwaltschaft angeklagt wurden ${ }^{38}$. $§ 266$ eröffnet der Staatsanwaltschaft allerdings die Möglichkeit einer Nachtragsanklage, d. h. der sofortigen und mündlichen Anklage der neuen prozessua- len Tat. Voraussetzung hierfür ist, dass das Gericht für die neue Tat zuständig ist und der Angeklagte ihrer Einbeziehung ins laufende Verfahren zustimmt. Selbst wenn diese Voraussetzungen geben sind, liegt es allerdings im Ermessen des S, ob er Nachtragsanklage erhebt oder ein neues Strafverfahren gegen A einleitet ${ }^{39}$.

Anmerkung: Das Zuständigkeitserfordernis ist jedenfalls erfüllt. Die örtliche Zuständigkeit ergibt sich aus $§ 13$ (Gerichtsstand des Sachzusammenhangs), da eine Person - nämlich der A - der Begehung mehrerer Taten beschuldigt wird, so dass ein persönlicher Zusammenhang zwischen ihnen besteht $t^{40}$. Die Tatsache, dass der Einschüchterungsversuch (für sich genommen) auch vor dem Einzelrichter hätte verhandelt werden können, erlaubt es dem Schöffengericht nicht, sich für unzuständig zu erklären $(\$ 269)$.

Frage 8: Welche Möglichkeiten hat A gegen das Urteil vorzugehen? (2 Punkte)

Urteile des Schöffengerichts können gem. $§ 312$ mit der Berufung angegriffen werden. Beruht das Urteil auf einer Rechtsverletzung kann A auch erwägen, statt der Berufung Sprungrevision gem. $\$ 335$ einzulegen.

36 Vgl. auch das Beispiel bei Volk Grundkurs StPO, 7. Aufl. 2010, § 13 Rdn. 6.

37 Schroeder/Verrel Strafprozessrecht, 5. Aufl. 2011, Rdn.270; Meyer-Goßner $\S 265$ Rdn. 2 m.w. N.

38 Beulke Strafprozessrecht, 11. Aufl. 2010, Rdn. 18; Kühne Strafprozessrecht, 8. Aufl. 2010, Rdn. 313; siehe auch Roxin/Schünemann Strafverfahrensrecht, 26. Aufl. 2009, § 13 Rdn. $1 \mathrm{ff}$.

39 Meyer-Goßner $§ 266$ Rdn. 4.

40 Vgl. hierzu auch Beulke Strafprozessrecht, 11. Aufl. 2010, Rdn. 46; MeyerGoßner §3 Rdn. 2; Volk Grundkurs StPO, 7. Aufl. 2010, § 13 Rdn. 10. 